\title{
Interplay between Gut Microbiome and Obesity: Insights from the Metabolic Syndrome, A Mini Review
}

\author{
Haukur Svansson ${ }^{1}$, Ilias Petrou ${ }^{1}$, Rida Abbasi ${ }^{1}$, Christian Skawran ${ }^{1}$, Iro Savvidou ${ }^{1}$, Stephanos \\ Christodoulides $^{1}$ and Ioannis Patrikios ${ }^{1 *}$ \\ ${ }^{1}$ School of Medicine, European University Cyprus, Cyprus
}

*Corresponding author: Ioannis Patrikios, School of Medicine, European University Cyprus, Cyprus

\section{ARTICLE INFO}

Received: 幽 February 19, 2020

Published: 蔧 March 04, 2020

Citation: Haukur S, Ilias P, Rida A, Christian S, Ioannis P. Interplay between Gut Microbiome and Obesity: Insights from the Metabolic Syndrome, A Mini Review. Biomed J Sci \& Tech Res 26(2)-2020. BJSTR. MS.ID.004324.

Keywords: Obesity; Metabolic Disease Syndrome; Microbiome; Lipopolysaccharides; Prebiotics; Probiotics; Symbiotic

\section{ABSTRACT}

Humans have a symbiotic relationship with gut microbiota, normally comprised of greater amount of the phyla firmicutes as opposed to Bacteroidetes. Which are respectfully categorized as gram-positive bacteria (GPB) and gram-negative bacteria (GNB). Their gut microbiome facilitates metabolism of substances which are otherwise indigestible to humans. Relevant literature reiterates their vital role to our daily life. Amongst individuals with metabolic disease syndrome (MetS) or/and obesity have shown to have disproportionate microbiome compared to healthy individuals. Giving rise to therapeutic options such as, prebiotics, probiotics and synbiotics as well as an educated diet. GPB, which have been shown to be a protective factor against lipopolysaccharide (LPS) induced gut-barrier dysfunctions and gut inflammation that are considered to be risk factors against MetS and obesity. In turn, the amount of GNB have a positive correlation with LPS. Relevant research supports dietary effects on microbiome and by extension on MetS and obesity. The authors acknowledge the need for more research and increased education to the general population, as well as to specialists about these implications.

\begin{abstract}
Abbreviations: LPS: Lipopolysaccharide; GNB: Gram-Negative Bacteria; GPB: Gram-Positive Bacteria; WHO: World Health Organization; LBP: LPS-Binding Protein; LDL-C: Low-Density Lipoprotein Cholesterol; HDL-C: High-Density Lipoprotein Cholesterol; VFA: Visceral Fat Area; FM: Fermented Milk; GOS: Galacto-Oligosaccharide; TC: Total Cholesterol; CFU: ColonyForming-Unit; TAG: Triacylglycerol; BFF: Botanical Fermented Food; BMI: Body Mass Index; MetS: Metabolic Disease Syndrome; LAB: Lactobacillus Bacteria
\end{abstract}

\section{Introduction}

In the animal kingdom, different lifeforms are frequently seen to develop evolutionary partnership, called symbiosis [1]. In some forms of these symbiotic relationships, one might be a reservoir inside the other which would be termed the host [1]. Humans are not excluded from the gain of symbiotic relationships [2]. The human body provides a stable environment for microorganisms to thrive in multiple places within the body [2]. In turn, the microbacteria facilitate functions otherwise impossible for humans [2]. It should be noted that, humans are not born with micro-bacteria, but require them through exposure [3]. Before discussing microbiota, it is crucial to acknowledge how the literature classifies them. Living things are categorized to a hierarchy of eight levels according to the study of taxonomy: Domain, Kingdom, Phylum, Class, Order, Family, Genus and Species [4]. Dependent on the lifeform being discussed, one applies different levels of specificity, dependent on known literature [4].

Regarding the bacteria literature, new discoveries are rapidly being made and a clear distinction between different species are 
commonly not available [4]. Thus, in the literature, it is common practice to refer to phyla, when more specificity is not possible [4]. Two of the most prominent human microbiota phyla are firstly the Firmicutes, accounting for $64 \%$ and categorized as GPB $[2,5]$. And secondly, Bacteroidetes, accounting for $23 \%$ of the human bacterial fauna, which are categorized as GNB. The GPB and GNB have shown a respectful association to either positive or negative effect to humans. Additionally, lipopolysaccharide (LPS), one of the building blocks in the outer membrane of GNB [6]. The role of LPS is to protect GNB against the hosts immune system, and thus a possible contributor to illness in the host [6]. Additionally, the death of GNB releases LPS in the gut, causing inflammation [7]. It should be noted that the intestinal epithelium of adults has a role to play in separating harmful and unharmful contents [8]. A dysfunction in the boundary layer, leads to a dysfunctional barrier between external and internal environment termed gut barrier dysfunction [8].

The complex role of the human gut microbiota, located in the gastrointestinal tract, operate on a wide spectrum [2]. Their effects are several including being a determinant of fatty acid synthesis [2], impacting the hosts cognitive functions [2]. Promoting intestinal mucosal integrity [2], providing essential nutrients such as vitamins and enzymes [2]. As well as protection against pathogens [2] and production of antimicrobial peptides [2]. Current scientific literature indicates the essential impact of the Gut microbiota [5-7]. The gut microbiota flora is a living entity, a variety of influencers can thus jeopardize its stability, leading to an instability termed "dysbiosis" [2]. These influencers include a sedentary lifestyle and an unhealthy diet. An individual's dysbiosis is a risk factor for a variety of metabolic syndromes, such as Metabolic Disease Syndrome (MetS) and obesity [9]. MetS is an umbrella term pertaining to a variety of risk factors for metabolic deficiency [10], and obesity is the excess of adipose tissue in one's body [11]. A tool commonly used to assess the state of adipose content, is the Body Mass Index (BMI) [12]. One is able to calculate the individual BMI, by dividing a person's weight in kilograms to their height in meters squared [12]. Where the following score intervals: $<18.5,(18,5$ to 24.9), (25.0 to 29,9), (30.0 to 34,9$)$ and (35.0 to 39,9) [12], get the respectful labels: Underweight, Normal, Overweight and Obese [13]. Additionally, the BMI z-score, is a value that adjusts for child age as well as sex [13].

When discussing the effects of nutrition, it is vital to acknowledge bioavailability. Bioavailability refers to the amount of nutrients stored and accessible [14]. A subcategory of bioavailability, bio-accessibility, which refers to the amount of nutrients released from the Gastrointestinal tract [14]. In turn bioactivity then refers to substance effect [14]. Food that is considered to have a positive effect beyond their nutrition, are termed functional food [15]. This group contains the subcategories: Probiotics, Prebiotics, and Synbiotics. Probiotics as defined by the World Health Organization
(WHO) are "Live microorganisms which when administered in adequate amounts confer a health benefit to the host" [15]. The WHO definition on prebiotics is "non-viable food component that confer health benefit (s) on the host associated with modulation of the microbiota" [15]. Synbiotics are then a combination of the two, where probiotics positively affect the probiotic organism(s) [15].

The above-mentioned measures have support in the literature as being successful in oral ingested therapies [16-24]. Conversely other micro-biotic therapeutics are available, such as in the form of fecal microbiota transplant, which could also serve as a screening factor with a high predictive value [2]

\section{Gut microbiome and Metabolic Disease Syndrome}

GNB are associated with LPS which increases gut permeability and therefore a higher amount of GNB is a clear indicating factor for MetS, obesity $[6,7]$ and gut barrier dysfunction. This increase in gut permeability is a result of several causes including alteration in the gut microbiota composition/activity. This can eventually lead to low-grade inflammation, MetS and obesity [22]. While not a novel syndrome, and considerably studied [10,25-27], recent literature suggests reappraisal of the MetS, as increasing literature supports the implications of gut microbiota to the MetS. Studies have demonstrated that there is a clear link between dietary consumption of specific food products, that lead to 'statistically significant changes in the composition of the gut microbiota in line with the prebiotic concept' [16]. Prebiotics selectively stimulate "the growth and/or activity of one or a limited number of bacteria in the colon and thus improves host health" [8]. Additionally, increasing evidence supports the notion that obesity is characterized by gut barrier changes, such as changes in the phyla and microbiota induced gut-membrane permeability, endotoxemia, which leads to disruption in the gut microbiota-host symbiotic relationship, suggesting a relationship between MetS and gut microbiota [8].

Studies have shown that obesity is associated with specific phyla, changes in the gut microbiota and with reduced bacterial diversity $[17,18]$. The literature has found that an increase of Firmicutes and Actinobacteria and a reduction of Bacteroidetes correlates and results in obesity [19]. This is further reflected by the proportions of Firmicutes, Actinobacteria and Bacteroidetes found in the feces of obese people $[20,17]$.

It has also been observed that there is an association between inflammatory gut tone with obesity [21]. The cause of this inflammatory gut tone is linked to LPS [21]. Studies have identified gut microbiota derived LPS as a key molecule in the early development of inflammation and metabolic diseases [22], again establishing a link between MetS and gut barrier dysfunctions. The gut microbiota can influence the host metabolism and contribute to the development of low-grade inflammation [22]. LPS is a powerful proinflammatory molecule from the cell wall of GNB and is continuously produced in the host gut with the death of GNB [22]. 
Whether MetS leads to the disproportions in microbiome, or vice versa, is an ongoing debate. What is clear is that we can effectively influence the microbiome and by extension MetS and obesity.

\section{Dietary effects}

MetS has been associated with diet in early life, known as the theory of "developmental origins of health and disease" by Barker in the 1990s [23]. Malnutrition during prenatal life is a risk factor for adulthood obesity and MetS [24-26]. Undernutrition leads to decrease in muscle mass, thus increasing risk of MetS [28]. Fat is abundant in our diet albeit not all fats are harmful $[16,29,30]$. Regarding our metabolic health, only trans and saturated fatty acids have shown to have adverse health effects $[28,29,30]$. The intake of fermented food and beverages like organic unpeeled unpasteurized fruits and vegetables, are associated with an improvement of bio accessibility and bioavailability of food bioactive components [31]. Sauerkraut, kimchi and Kefir are powerful probiotic foods as they contribute a diverse array of microorganisms [31].

Research suggests regular consumption of these fermented foods can have a direct positive influence on the gut microbiota by passing through the gut transiently and if regularly consumed, surpass undesirable microbes, and thus the potential to affect health [32]. Botanical Fermented food (BFF), contain abundant microbiota, they are rich in carbohydrates which are easily accessible to microbiota. In the scientific literature, several reviews have been conducted on the health promoting factors of BFF [33], but as of this writing, no such review has been published regarding the potential of BFF to MetS [33]. Regarding inflammatory diseases such as those that contribute to MetS, GNB contributes to systemic and local LPS burden [34]. Chronic exposure to LPS induces features of MetS and interacts with the innate immune system, through LPS-binding protein (LBP) and its co-receptor CD14 [35]. Higher levels of LPS occur in healthy people after a high fat meal [36]. Though obese are observed having higher levels of circulating LPS in fasting and fed state [36]. Chronic exposure to elevated LPS has been shown to correlate with abdominal obesity and glycemic control [37]. Thus, indicating a potential role of the innate immune system in MetS [35].

\section{Therapeutics in Obesity: Probiotics, Prebiotics, and Synbiotics}

The literature has shown that overweight individuals can possibly manage their weight through a variety of therapeutics, pertaining gut microbiota [38]. As previously stated, the gut microbiota composition is an influencing factor of obesity [38], furthermore the microbiome can be modified with the use of probiotics, prebiotics, and synbiotics [39]. Several probiotics including Lactobacillus bacteria (LAB) (e.g., L. Casei strain Shirota LAB13, L. Gasseri, L. Rhamnosus, L. Plantarum) and Bifidobacterium (e.g., B. Infantis, B. Longum, and B. Breve B3) [5]. LAB is found in carbohydrate-containing substrates, such as the mucosal membrane of vertebrates, in plant, sewage, fermented foods [3]. Supplementation of capsules with bifidobacteria, lactobacilli, and S. Thermophilus have shown a significant improvement in the overweight individual 's lipid profiles [40]. Reducing total cholesterol (TC), triacylglycerol (TAG), the simplest lipids formed by fatty acids, low-density lipoprotein cholesterol (LDL-C) levels and increasing high-density lipoprotein cholesterol (HDL-C) levels [40].

The effect of a specific probiotic called L. gasseri SBT2055 (LG2055) was examined in a study using adults with large visceral fat area (VFA) [41]. The participants were assigned to receive either fermented milk (FM) containing LG2055 or FM without LG2055 for 12 weeks [41]. The results using the probiotic LG2055 showed lowering effects on abdominal adiposity and body weight [41]. In general, the supplementation of selected probiotics appears to have an important impact on BMI, VFA and hip circumference [38]. Prebiotics consist of complex carbohydrates (one or more) such as, insulin, fructo-oligosaccharide (FOS), or galacto-oligosaccharide (GOS) which can be used as substrates for the microbiota. [42,39]. The supplementation of oligofructose in a high fat diet has been shown to increases the number of intestinal Bifidobacterium and LAB and reduce obesity [39]. The authors recommend advocation of better public awareness regarding the availability of prebiotics in food and their positive effects, as presented in the literature [43]. Examples of prebiotic food sources include yoghurt, cottage cheese, apple cider vinegar, vinegar and miso [43].

Different synbiotics including L. rhamnosus CGMCC1.3724, $6 \times 108$ Colony-forming-unit (CFU), and inulin [44]/ L. casei, L. rhamnosus, S. thermophilus, B. breve, L. acidophilus, B. longum, L. bulgaricus, and FOS [45]/ L. acidophilus, L. rhamnosus, B. bifidum, B. longum, E. faecium, and FOS [46]. A therapy with synbiotics can reduce BMI z-score, body fat located inside the peritoneal cavity, and waist circumference in children [13], as well as TC, LDL-C, the main source of cholesterol buildup and blockage in the arteries [47], and TAG and serum levels [38].

\section{Conclusion}

Research indicates that microbiome dysbiosis is a predisposition for MetS and obesity [6,7,8,22,]. With increasing evidence in the literature, the public and health specialists should be educated accordingly about the implications of the microbiome. Supplementation in the form of probiotics, prebiotics and synbiotics, has ample evidence in the literature for being a protective factor against dysbiosis [38-46]. At the same time, the authors acknowledge a knowledge barrier, in terms of inaccuracy in taxonomy. As some micro-bacteria can only be reduced to a level of phyla, due to research gaps [4]. Leaving ample space for improvement. Authors conclude by addressing the need for more research in this area [4]. 


\section{References}

1. Hedayat K, Lapraz J (2019) Symbiosis. The Theory of Endobiogeny 6375.

2. Srikanth P, Pushpanathan P, Mathew G, Selvarajan S, Seshadri K, et al. (2019) Gut microbiota and its mysteries. Indian Journal of Medical Microbiology 37(2): 268-277.

3. De Vrese M, Schrezenmeir J (2008) Probiotics Prebiotics and Synbiotics. Food Biotechnology 111: 1-66.

4. Tyler A, Smith M, Silverberg M (2014) Analyzing the Human Microbiome: A "How To" guide for Physicians. American Journal of Gastroenterology 109(7): 983-993.

5. Abenavoli L, Scarpellini E, Colica C, Boccuto L, Salehi B, et al. (2019) Gut Microbiota and Obesity: A Role for Probiotics. Nutrients 11(11): 2690.

6. (2019) Lipopolysaccharide and Its Constituents Encyclopedia.com.

7. Cani P, Delzenne N (2009) The Role of the Gut Microbiota in Energy Metabolism and Metabolic Disease. Current Pharmaceutical Design 15(13): 1546-1558.

8. Bording Jorgensen M, Alipour M, Wine E, Danesh G (2017) Inflammasome Activation by ATP Enhances Citrobacter rodentium Clearance through ROS Generation. Cellular Physiology and Biochemistry 41(1): 193-204.

9. Davani Davari D, Negahdaripour M, Karimzadeh I, Seifan M, Mohkam M, et al. (2019) Prebiotics: Definition Types Sources Mechanisms and Clinical Applications. Foods 8(3): 92.

10. Dabke K, Hendrick G, Devkota S (2019) The gut microbiome and metabolic syndrome. Journal of Clinical Investigation 129(10): 40504057

11. Goossens G (2017) The Metabolic Phenotype in Obesity: Fat Mass, Body Fat Distribution, and Adipose Tissue Function. Obesity Facts 10(3): 207215.

12. R Balentine J (2019) Obesity Definition Charts BMI Causes Treatment.

13. Must A, Anderson S (2006) Body mass index in children and adolescents: considerations for population-based applications. International Journal of Obesity 30(4): 590-594.

14. Galanakis C (2019) What is the Difference Between Bioavailability Bioaccessibility and Bioactivity of Food Components? SciTech Connect.

15. Manigandan T, Mangaiyarkarasi S, Hemalatha R, Hemalatha V, Murali N, et al. (2012) Probiotics, Prebiotics and Synbiotics - A Review. Biomedical \& Pharmacology Journal 5(2): 295-304.

16. Sáez Lara M, Robles Sanchez C, Ruiz Ojeda F, Plaza Diaz J, Gil A, et al. (2016) Effects of Probiotics and Synbiotics on Obesity, Insulin Resistance Syndrome, Type 2 Diabetes and Non-Alcoholic Fatty Liver Disease: A Review of Human Clinical Trials. International Journal of Molecular Sciences 17(6): 928.

17. De Clercq N, Groen A, Romijn J, Nieuwdorp M (2016) Gut Microbiota in Obesity and Undernutrition. Advances in Nutrition: An International Review Journal 7(6): 1080-1089.

18. Rajkumar H, Mahmood N, Kumar M, Varikuti S, Challa H, et al. (2014) Effect of Probiotic (VSL\#3) and Omega-3 on Lipid Profile, Insulin Sensitivity, Inflammatory Markers, and Gut Colonization in Overweight Adults: A Randomized, Controlled Trial. Mediators of Inflammation 2014: 1-8.

19. Kadooka Y, Sato M, Imaizumi K, Ogawa A, Ikuyama K, et al. (2010) Regulation of abdominal adiposity by probiotics (Lactobacillus gasseri SBT2055) in adults with obese tendencies in a randomized controlled trial. European Journal of Clinical Nutrition 64(6): 636-643.

20. Chu J, Kang S, Kim S, Lee S, Lee Y, et al. (2019) Prebiotic UG1601 mitigates constipation-related events in association with gut microbiota: A randomized placebo-controlled intervention study. World Journal of Gastroenterology 25(40): 6129-6144.
21. Chan S, Hawley C, Campbell K, Morrison M, Campbell S, et al. (2019) Transplant associated infections-The role of the gastrointestinal microbiota and potential therapeutic options. Nephrology 25(1): 5-13

22. Sanchez M, Darimont C, Drapeau V, Emady Azar S, Philippe L, et al. (2013) Effect of Lactobacillus Rhamnosus CGMCC1.3724 Supplementation on Weight loss and Maintenance in Obese Men and Women. Canadian Journal of Diabetes 37: S269.

23. Safavi M, Farajian S, Kelishadi R, Mirlohi M, Hashemipour, et al. (2013) The effects of synbiotic supplementation on some cardio-metabolic risk factors in overweight and obese children: A randomized triple-masked controlled trial. International Journal of Food Sciences and Nutrition 64(6): 687-693

24. Ipar N, Aydogdu S, Yildirim G, Inal M, Gies I, et al. (2015) Effects of synbiotic on anthropometry, lipid profile and oxidative stress in obese children. Beneficial Microbes 6(6): 775-781.

25. Roberfroid M, Gibson G, Hoyles L, McCartney A, Rastall R, et al. (2010) Prebiotic effects: metabolic and health benefits. British Journal of Nutrition 104(S2): S61-S63.

26. Waldram A, Holmes E, Wang Y, Rantalainen M, Wilson I, et al. (2009) Top-Down Systems Biology Modeling of Host Metabotype Microbiome Associations in Obese Rodents. Journal of Proteome Research 8(5): 2361-2375.

27. Kalliomäki M, Carmen Collado M, Salminen S, Isolauri E (2008) Early differences in fecal microbiota composition in children may predict overweight. The American Journal of Clinical Nutrition 87(3): 534-538.

28. Brown L (2014) Endocrine regulation of fetal skeletal muscle growth: Impact on future metabolic health. Journal of Endocrinology 221(2): R13-R29.

29. Turnbaugh P, Hamady M, Yatsunenko T, Cantarel B, Duncan A, et al. (2008) A core gut microbiome in obese and lean twins. Nature 457(7228): 480-484

30. Ley R, Backhed F, Turnbaugh P, Lozupone C, Knight R, et al. (2005) Obesity alters gut microbial ecology. Proceedings of the National Academy of Sciences 102(31): 11070-11075.

31. Plovier H, Cani P (2017) Microbial Impact on Host Metabolism: Opportunities for Novel Treatments of Nutritional Disorders? Microbiology Spectrum 5(3)

32. Everard A, Cani P (2013) Diabetes, obesity and gut microbiota. Best Practice \& Research Clinical Gastroenterology 27(1): 73-83.

33. Knip M, Åkerblom H (2005) Early Nutrition and Later Diabetes Risk. Advances in Experimental Medicine and Biology 569: 142-150.

34. Desai M, Jellyman J, Ross M (2015) Epigenomics, gestational programming and risk of metabolic syndrome. International Journal of Obesity 39(4): 633-641.

35. Tarrade A, Panchenko P, Junien C, Gabory A (2015) Placental contribution to nutritional programming of health and diseases: Epigenetics and sexual dimorphism. Journal of Experimental Biology 218(1): 50-58.

36. Brenseke B, Prater M, Bahamonde J, Gutierrez J (2013) Current Thoughts on Maternal Nutrition and Fetal Programming of the Metabolic Syndrome. Journal of Pregnancy 2013: 1-13.

37. Calton E, James A, Pannu P, Soares M (2014) Certain dietary patterns are beneficial for the metabolic syndrome: Reviewing the evidence. Nutrition Research 34(7): 559-568.

38. Davì G, Santilli F, Patrono C (2010) Nutraceuticals in Diabetes and Metabolic Syndrome. Cardiovascular Therapeutics 28(4): 216-226.

39. Roche H (2005) Fatty acids and the metabolic syndrome. Proceedings of the Nutrition Society 64(1): 23-29.

40. JF, VB, VC, LP, MP, et al. (2019) Impact of Mushroom Nutrition on Microbiota and Potential for Preventative Health. 
41. Mouw M, Team G, Prados A, Sáez C (2019) The unique properties and health benefits of fermented foods. Gut Microbiota for Health.

42. Chan M, Baxter H, Larsen N, Jespersen L, Ekinci E, et al. (2019) Impact of botanical fermented foods on metabolic biomarkers and gut microbiota in adults with metabolic syndrome and type 2 diabetes: A systematic review protocol. BMJ Open 9(7): e029242.

\section{ISSN: 2574-1241}

DOI: 10.26717/BJSTR.2020.26.004324

Ioannis Patrikios. Biomed J Sci \& Tech Res

(C) (P) This work is licensed under Creative

Submission Link: https://biomedres.us/submit-manuscript.php
43. Berding K, Donovan S (2016) Microbiome and nutrition in autism spectrum disorder: Current knowledge and research needs. Nutrition Reviews 74(12): 723-736.

44. Awoyemi A, Trøseid M, Arnesen H, Solheim S, Seljeflot I, et al. (2018) Markers of metabolic endotoxemia as related to metabolic syndrome in an elderly male population at high cardiovascular risk: A cross-sectional

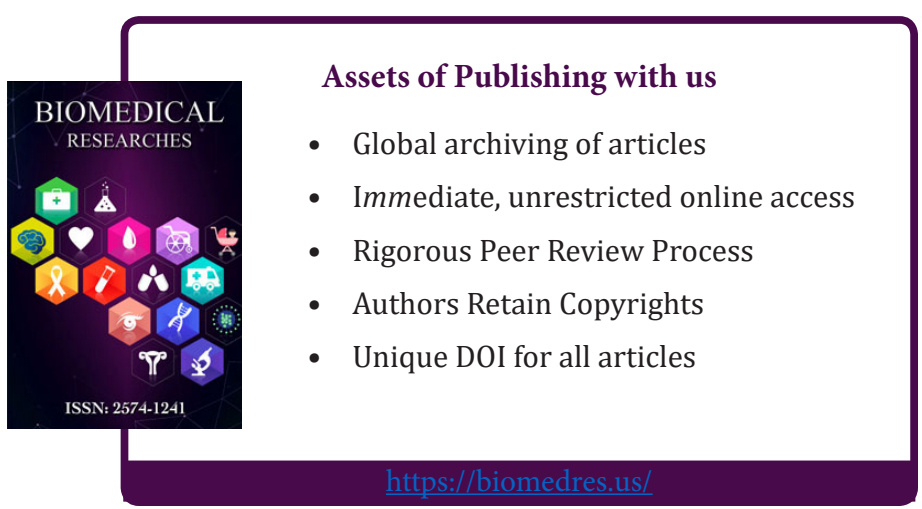

